\title{
Wide Frequency Range AC Magnetic Properties of Fe-Based Composite Materials
}

\author{
P. KollâR ${ }^{a}$, Z. BirČÁkováa ${ }^{a}$, J. FÜzer $^{a}$, J. FÜzerová ${ }^{b}$, R. BureŠ ${ }^{c}$ And M. Fáberová ${ }^{c}$ \\ ${ }^{a}$ Institute of Physics, Faculty of Science, P.J. Šafárik University, Park Angelinum 9, 04154 Košice, Slovakia \\ ${ }^{b}$ Department of Applied Mathematics, Faculty of Mechanical Engineering, Technical University in Košice
}

04200 Košice, Slovakia

${ }^{c}$ Institute of Materials Research, Slovak Academy of Sciences, Watsonova 47, 04001 Košice, Slovakia

The aim of this work was to analyze the influence of resin content on contribution of the hysteresis, eddy current and anomalous losses to the total losses in the frequency range from DC to $150 \mathrm{kHz}$. The samples of composite material were prepared in the form of the ring with outer diameter of $25 \mathrm{~mm}$, inner diameter of $17 \mathrm{~mm}$ and height approximately $3 \mathrm{~mm}$ and in the form of cylinder with diameter of $25 \mathrm{~mm}$ and height approximately $3 \mathrm{~mm}$ by a compaction of mixture of iron powder ASC100.29 (90 vol.\%) and commercial termoset resins. The DC hysteresis losses were obtained by the measurements of DC hysteresis loops and the total power losses in the frequency range $0.4 \mathrm{~Hz}-150 \mathrm{kHz}$ from $\mathrm{AC}$ hysteresis loops, both at maximum induction $0.05,0.1$ and $0.2 \mathrm{~T}$. The magnetic properties of the composite rings were compared with the properties of the material prepared from the powder supported by Höganäs AB Sweden. By analyzing the frequency dependence of total losses of the Fe-based $\mathrm{SMC}$ we found out that hysteresis losses contribute to the total losses as a majority component.

PACS numbers: 81.05.Ni, 85.70.-w, 75.60.-d, 75.50.Bb

\section{Introduction}

Soft magnetic composites (SMC, ferromagnetic powder particles surrounded by an electrical insulating film) is a well known group of materials exhibiting very good soft magnetic properties as low hysteresis losses, low eddy current losses, high permeability at low magnetic field and high saturation flux density $[1,2]$. These materials are suitable for various $\mathrm{AC}$ and $\mathrm{DC}$ electromagnetic applications (cores with three-dimensional isotropic ferromagnetic behaviour for transformers, electromotors and electromagnetic circuits, sensors, actuators, low frequency filters, induction field coils, magnetic seal systems and magnetic field shielding) [3]. Many scientists think that the explorations possibilities of SMC are still not exhausted and forecast further progress leading to the replacement of electrical steel sheets or ferrites in some applications by these composites [3].

The aim of this work was to investigate the influence of resin content on $\mathrm{AC}$ magnetic properties of Fe-based composite material to extend possibilities for application of this kind of material at higher frequency.

\section{Samples preparation}

The SMC samples were prepared by conventional powder metallurgy in form of a ring (for magnetic measurements) and in form of a cylinder (for electric resistivity measurements) [4]. The pure iron powder ASC 100.29, Höganäs AB Sweden [5] was chosen as a ferromagnetic material and a phenol-formaldehyde resin (Bakelite ATM) as an insulator. The particles of the iron powder exhibit (according to the material producer) following particle size distribution: $50 \mathrm{wt} \%$ particles have the diameter below $75 \mu \mathrm{m}$ and $0.9 \mathrm{wt} \%$ - above $180 \mu \mathrm{m}$.
The bakelite was milled for $3 \times 30 \mathrm{~s}$ in a knife mill to obtain the particle size below $100 \mu \mathrm{m}$. The experimental samples were prepared by wet homogenisation of 90 vol.\% of iron (sample A) and resin with acetone.

The homogenised powder was pressed in a cylindrical die at an uniaxial pressure to obtain compacts in the form of cylinder or ring.

After that the resulting product was cured at a temperature of $165^{\circ} \mathrm{C}$ for $60 \mathrm{~min}$ in an electric furnace in an air atmosphere.

The thermoset phenol-formaldehyde resin covers the iron powder particles, the resin electrically insulates conductive iron particles and creates the mechanical bond after curing [4].

Sample $S$ is a reference material, prepared from Somaloy ${ }^{\circledR} 700$ powder (covered by insulating film) provided by Höganäs AB, Sweden by producer-developed technology (compaction at $800 \mathrm{MPa}$ and heat treatment at $530^{\circ} \mathrm{C}$ for $30 \mathrm{~min}$ in air). Parameters of the samples are in Table.

\section{Experimental}

The structure of the samples was investigated by scanning electron microscopy (TESLA BS 340). The hysteresis losses were calculated from the minor DC hysteresis loops (maximum flux density of 0.05, 0.1 and $0.2 \mathrm{~T}$ ) measured by a fluxmeter based hysteresisgraph. AC hysteresis loops were measured by three different $\mathrm{AC}$ hysteresisgraphs: in the frequency range $0.4-50 \mathrm{~Hz}$ by a fluxmeter based hysteresisgraph, in the frequency range $50 \mathrm{~Hz}-1 \mathrm{kHz}$ by MATS-2010M and in the frequency range $1-150 \mathrm{kHz}$ by MATS-2010SA at the maximum flux density of $0.05,0.1$ and $0.2 \mathrm{~T}$. The total losses $W_{\mathrm{t}}$ were calcu- 
Parameters of the samples.

TABLE

\begin{tabular}{l|c|c}
\hline \hline \multicolumn{1}{c|}{ Sample } & $A$ & $S$ \\
\hline volume fraction of Fe [\%] & 90.0 & 98.7 \\
outer diameter [mm] & 24.00 & 24.10 \\
inner diameter [mm] & 17.87 & 17.71 \\
height [mm] & 2.75 & 2.81 \\
mass [g] & 3.439 & 3.870 \\
density [g/cm ${ }^{3}$ ] & 5.22 & 6.57 \\
compaction pressure [MPa] & 600 & 800 \\
compaction temperature [ $\left.{ }^{\circ} \mathrm{C}\right]$ & 165 & 530 \\
porosity [\%] & 13.6 & 12.4 \\
specific resistivity $[\mu \Omega \mathrm{m}]$ & 505 & 4.58 \\
hysteresis loss $\left[\mathrm{J} / \mathrm{m}^{3}\right.$ ] at $0.05 \mathrm{~T}^{a}$ & 7 & 4 \\
hysteresis loss $\left[\mathrm{J} / \mathrm{m}^{3}\right.$ ] at $0.1 \mathrm{~T}^{a}$ & 31 & 17 \\
hysteresis loss $\left[\mathrm{J} / \mathrm{m}^{3}\right.$ ] at $0.2 \mathrm{~T}^{a}$ & 1077 & 67 \\
\hline
\end{tabular}

${ }^{a}$ experimental values

lated directly from measured hysteresis loops. The peak relative permeability was calculated from hysteresis loops measured in the frequency range $1-150 \mathrm{kHz}$ at maximum flux density of $0.05 \mathrm{~T}$.

The specific electrical resistivity of the composite material in the form of the cylinder with diameter of about $24 \mathrm{~mm}$ and from $2 \mathrm{~mm}$ to $4 \mathrm{~mm}$ height was measured by the van der Pauw method (assuming to be isotropic in the plane parallel to the cylinder base) [6].

\section{Power losses}

The total energy losses $W_{\mathrm{t}}$ (in units $\mathrm{J} / \mathrm{m}^{3}$ ) consist of hysteresis $W_{\mathrm{h}}$, eddy current $W_{\mathrm{e}}$ and anomalous $W_{\mathrm{a}}$ [7-10]:

$$
W_{\mathrm{t}}=W_{\mathrm{h}}+W_{\mathrm{e}}+W_{\mathrm{a}} .
$$

Hysteresis losses $W_{\mathrm{h}}$ are assumed to be frequency independent and can be obtained by extrapolation the total losses frequency dependence to $f=0$.

Other possibility how to characterize the frequency dependence of the power dissipation in a magnetic material during the time of $1 \mathrm{~s}$ is to use quantity $P_{\mathrm{t}}$ (unit $\mathrm{W} / \mathrm{kg}$ ) by multiplication of Eq. (1) by the factor $f / \rho(f-$ frequency, $\rho$ - density of the material)

$$
P_{\mathrm{t}}=W_{\mathrm{h}} \frac{f}{\rho}+P_{\mathrm{e}}+P_{\mathrm{a}} .
$$

The eddy current losses $P_{\mathrm{e}}$ can be expressed according to $[7,8]$ by

$$
P_{\mathrm{e}}=\frac{\left(\pi d B_{\mathrm{m}} f\right)^{2}}{\beta \rho_{\mathrm{R}} \rho},
$$

where $d$ is effective dimension (for the material prepared by casting $d$ is thickness of the sample, for laminated material, thickness of the sheet), $B_{\mathrm{m}}$ is maximum flux density, $f-$ frequency, $\rho_{\mathrm{R}}-$ specific resistivity, $\rho-$ density of the material and $\beta$ - geometrical coefficient. For rectangular cross-section perpendicular to the direction of magnetic flux is

$$
\beta=\frac{6}{1-0.633(w / h) \tanh (1.58 h / w)},
$$

where $w$ is width and $h$ is height of the rectangle [7].

The anomalous contribution at sinusoidal magnetic flux in the material can be expressed as

$$
P_{\mathrm{a}}=8.8 \sqrt{\sigma G S V_{0}}\left(B_{\mathrm{m}} f\right)^{3 / 2},
$$

where $\sigma$ is specific electric conductivity of the material, $S$ is cross-section of the material perpendicular to the magnetic flux, $B_{\mathrm{m}}$ - maximum flux density, $G$ and $V_{0}$ - parameters depending on the material and magnetization [7].

\section{Results}

The hysteresis losses of both samples calculated from DC hysteresis loops are presented in Table. The total losses dependences as a function of frequency in the frequency range $0.4 \mathrm{~Hz}-150 \mathrm{kHz}$ at maximum flux density $B_{\mathrm{m}}$ of $0.05,0.1$ and $0.2 \mathrm{~T}$ are depicted in Fig. 1 .

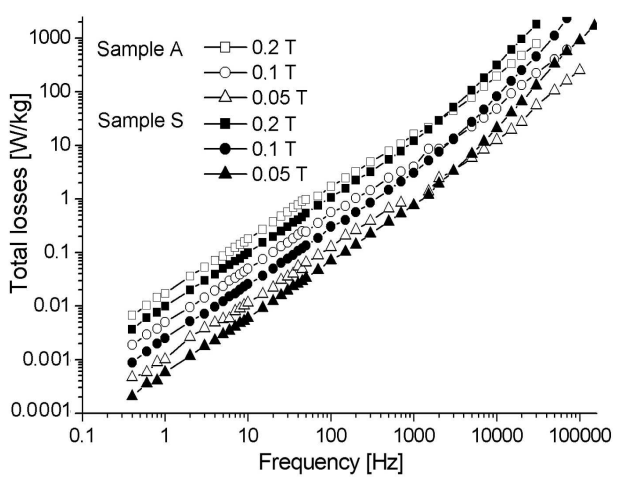

Fig. 1. Total losses $P_{\mathrm{t}}$ as a function of the frequency $f$ for the sample $A$ and $S$ measured at maximum flux density $B_{\mathrm{m}}=0.05,0.1$ and $0.2 \mathrm{~T}$ in frequency range $0.4 \mathrm{~Hz}-150 \mathrm{kHz}$.

The values of total losses as a function of the frequency of the sample $A$ increase less steeply than that for samples $S$. We assume that lower slope of total losses dependence of the sample $A$ is caused by the lower contribution of the eddy current losses to the total losses due to higher specific resistivity (approximately 100 times) of this sample in comparison with the specific resistivity of the sample $S$, Table.

The contribution of the anomalous losses $W_{\mathrm{a}}$ (according to Eq. (5) $W_{\mathrm{a}} \sim f^{1 / 2}$ ) to the total losses $W_{\mathrm{t}}$ is best observable at low frequency range. In order to detect this contribution we have plotted the total losses in frequency range below $50 \mathrm{~Hz}$. The losses plotted in Fig. 2 for frequency " $0 \mathrm{~Hz}$ " is hysteresis loss $W_{\mathrm{h}}$. The fraction of ferromagnetic material (Fe) is larger in sample $S$, that is why the magnetic contact between ferromagnetic particles is better in this sample, which leads to the lower values of hysteresis loss in comparison with sample $A$ containing relatively large amount of resin.

The peak relative permeability of the sample $S$, reflecting the slope of the hysteresis loop, is higher than that for sample $A$, Fig. 3 . 


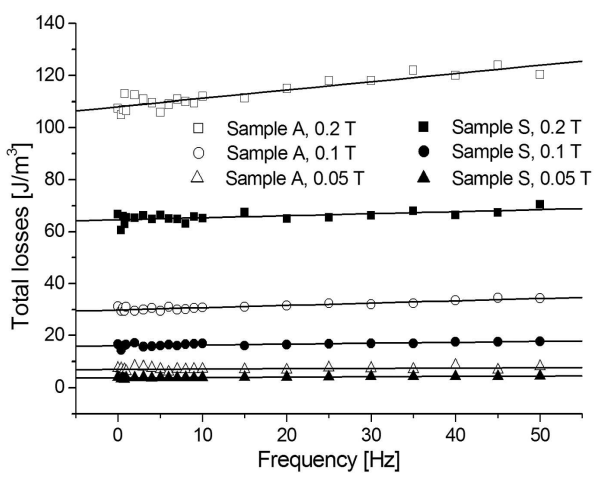

Fig. 2. Total losses $W_{\mathrm{t}}$ as a function of the frequency $f$ for the sample $A$ and $S$ measured at maximum flux density $B_{\mathrm{m}}=0.05,0.1$ and $0.2 \mathrm{~T}$ in frequency range $0.4-50 \mathrm{~Hz}$.

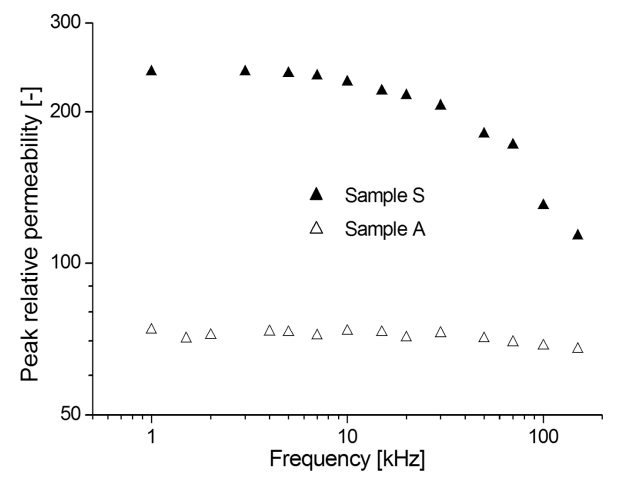

Fig. 3. Peak relative permeability $\mu_{\mathrm{p}}$ as a function of the frequency $f$ for the sample $A$ and $S$ calculated from $\mathrm{AC}$ hysteresis loops measured at maximum flux density $B_{\mathrm{m}}=0.05 \mathrm{~T}$ in frequency range $1-150 \mathrm{kHz}$.

The sample $A$ containing larger fraction of nonferromagnetic material exhibits larger value of the inner demagnetization factor $N[11,12]$. The iron powder particles create demagnetization field (which is ruled by the mean distance between a pair of ferromagnetic particles), oriented opposite to the external magnetic field $H$, causing the decrease of the internal magnetic field $H_{\text {int }}$ [7]:

$$
H_{\text {int }}=H-N M \text {, }
$$

where $M$ is magnetization of the powder particle.

We suppose that the decrease of the peak relative permeability of the sample $S$ with frequency is caused by the magnetic viscosity of the material [7]. The frequency dependence of total losses of composite material in comparison with metallic compacted powder $\mathrm{Fe}-\mathrm{Ni}$ based material exhibits lower values and lower slope, which confirms that composite materials are more suitable for high frequency application than metallic materials [13].

\section{Conclusion}

From the investigation of DC and AC magnetic properties of composite material based on iron powder (90 vol.\%) and phenol-formaldehyde resin (10 vol.\%), as insulator we found that the frequency dependence of total losses increases slower than that for reference Somaloy ${ }^{\circledR}$ 700 powder based sample. It is caused by the lower contribution of eddy current losses of composite material in which the contribution of hysteresis losses is dominant. The analysis of the total losses for both samples in low frequency range shows no significant contribution of anomalous losses. The frequency dependence of peak relative permeability of the composite material (which reflects the mean distance between a pair of ferromagnetic particles) is lower but practically constant in the whole frequency range $1-150 \mathrm{kHz}$ in comparison with that for reference Somaloy ${ }^{\circledR} 700$ material which steeply decreases with the increase of frequency.

\section{Acknowledgments}

This work was realized within the frame of the project "Centre of Excellence of Advanced Materials with Nanoand Submicron-Structure", which is supported by the Operational Program "Research and Development" financed through European Regional Development Fund ITMS: 26220120019. This work was also supported by the Slovak Research and Development Agency under the contract No. APVV-0490-07 MICOMAT and by the Scientific Grant Agency of the Ministry of Education of Slovak Republic and the Slovak Academy of Sciences, project No. VEGA 1/0311/10 and No. VEGA 2/0149/09. Special thanks to Höganäs AB Sweden for providing Somaloy ${ }^{\circledR}$ powder.

\section{References}

[1] H. Shokrollahi, K. Janghorban, J. Mater. Proc. Technol. 189, 1 (2007).

[2] A.H. Taghvaei, H. Shokrollahi, K. Janghorbana, J. Alloys Comp. 481, 681 (2009).

[3] T. Maeda, H. Tyoda, N. Igarashi, K. Hirose, K. Mimura, T. Nishioka, A. Ikegaya, SEI Tech. Rev. 60, 3 (2005).

[4] P. Kollár, J. Füzer, R. Bureš, M. Fáberová, IEEE Trans. Magn., 46, 467 (2010).

[5] Product catalog: Somaloy ${ }^{\circledR}$ Metal Powders for SMC Components, Höganäs AB, Sweden.

[6] L.J. van der Pauw, Philips Techn. Rev. 20, 220 (1958).

[7] R.M. Bozorth, Ferromagnetism, 3rd ed., IEEE Press, Piscataway, NJ 1993.

[8] G. Bertotti, Hysteresis in Magnetism, Academic Press, San Diego 1998.

[9] T.D. Shen, U. Harms, R.B. Schwarz, Mater. Sci. Forum 386-388, 441 (2002).

[10] H. Chiriac, N. Lupu, M. Tibu, IEEE Trans. Magn. 39, 2040 (2003).

[11] J.-L. Mattei, M. Le Floc'h, J. Magn. Magn. Mater. 257, 335 (2003).

[12] M. Anhalt, B. Weidenfeller, J.-L. Mattei, J. Magn. Magn. Mater. 320, e844 (2008).

[13] P. Kollár, D. Olekšáková, J. Füzer, J. Kováč, S. Roth, K. Polański, J. Magn. Magn. Mater. 310, 2609 (2007). 